\title{
汽车磁流变液制动器温度特性仿真与试验研究*
}

\author{
王道明 ${ }^{1}$ 姚 兰 ${ }^{1}$ 邵文涁 ${ }^{1,2}$ 訾 斌 ${ }^{1}$ 陈无畏 ${ }^{1}$ \\ (1. 合肥工业大学机械工程学院 合肥 230009; \\ 2. 安徽江淮汽车集团股份有限公司 合肥 230601)
}

\begin{abstract}
摘要: 针对磁流变液制动器工作时内部热量聚集造成其制动性能下降问题, 采用仿真分析与试验验证相结合的方法对其温度 特性进行研究。首先分析计算磁流变液制动器的热量来源和生热率, 在此基础上建立其温度场数学模型; 其次分别针对汽车 正常制动、紧急制动和频繁间隙制动等三种不同制动工况, 进行磁流变液制动器的瞬态温度场仿真分析; 最后搭建汽车磁流 变液制动器试验平台开展输出制动力、制动性能和温度特性的试验研究。结果表明: 在相同线圈电流下, 磁流变液制动器表 现出良好的恒减速度制动特性; 不同制动工况下一个制动周期内工作间隙处的温度均呈现先迅速增大后逐渐降低的变化过 程, 并且制动初速度和线圈电流越大, 温升幅度和速率均越大; 整个制动周期内测点处温度的试验值与仿真值在数值和趋势 上较为吻合，表明所建立的温度场仿真模型能够较好地反映磁流变液制动器实际制动过程的温度特性。
\end{abstract}

关键词: 磁流变液制动器; 温度特性; 仿真分析; 制动试验

中图分类号: TH139

\section{Simulation and Experimental Study on Temperature Characteristics of Magnetorheological Fluid Brake for Vehicles}

\author{
WANG Daoming $^{1} \quad$ YAO Lan $^{1} \quad$ SHAO Wenbin ${ }^{1,2}$ ZI Bin ${ }^{1} \quad$ CHEN Wuwei ${ }^{1}$ \\ (1. School of Mechanical Engineering, Hefei University of Technology, Hefei 230009; \\ 2. Anhui Jianghuai Automobile Group Corp., Ltd., Hefei 230601)
}

\begin{abstract}
In view of the problem that the braking performance is decreased due to the heat accumulation in the braking process of the magnetorheological fluid brake (MRB), simulation analysis and experimental evaluation of temperature characteristics of MRB are performed. Based on the analysis and calculation of the heat source and heat generation rate of MRB, a mathematical model for the temperature field is established. Then, aiming at three different braking conditions for vehicles: normal braking, emergency braking and frequency interval braking, the transient temperature field of MRB is simulated and analyzed. Finally, an experimental platform is developed and several braking tests are carried out to evaluate the output braking torque, the braking performance and the temperature characteristic of MRB. Results show that the MRB exhibits a good constant deceleration braking property under a same coil current. During a braking period, the temperatures at the working gap first increase and then decrease under various conditions. Test results and simulation values for the temperature at the measuring point are in good agreement, which indicates that the established temperature simulation model can reflect quite well the actual temperature characteristic of the MRB.
\end{abstract}

Key words: magnetorheological fluid brake; temperature characteristic; simulation analysis; braking test

\section{0 前言}

制动器作为汽车制动系统的执行元件, 其性能 优劣对于汽车的安全行驶起着极其重要的作用。目 前普遍使用的机械摩擦式制动器, 存在制动效能低、 动作滞后、热衰退、磨损严重和制动时汽车方向不

* 国家自然科学基金(51505114)、中国博士后科学基金特别(2016T90561) 和安徽省自然科学基金(1608085QE116)资助项目。20181203 收到初稿, 20190201 收到修改稿
稳定等问题 ${ }^{[1]}$ 。随着汽车发展趋向智能化、电子化, 迫切需要一种结构简单、适用新型线控技术且控制 性能优越的汽车制动器解决方案。

当前, 智能材料的飞速发展为汽车制动系统的 完善提供了良好契机 ${ }^{[2]}$ 。磁流变液制动器以磁流变 液为制动介质, 以调节电流为控制手段, 可实现运 动机械的可控柔性制动。得益于磁流变效应响应快 (毫秒级)、控制简单、能耗低和工作部件磨损小等 特点 ${ }^{[3]}$, 采用磁流变液制动器作为汽车制动执行器 可有效提高制动灵敏性、缩短制动距离; 通过配合 
信息反馈系统, 保证制动力实时匹配路面附着条件 变化, 实现连续、精准、无脉动的 $\mathrm{ABS}$ 制动, 提高 制动柔顺性和制动效能; 可与车载电子控制系统无 缝结合, 实现协同控制 ${ }^{[4]}$ 。因此, 开展汽车磁流变 液制动器的研究对于提高制动安全性和舒适性具有 重要理论意义和实际应用价值 ${ }^{[5]}$ 。

然而, 在汽车制动过程中, 制动界面间磁流变 液颗粒链间摩擦产生热量聚集, 导致磁流变液传力 特性下降, 对制动性能造成不利影响 ${ }^{[6-7]}$ 。因此, 开 展磁流变液制动器温度特性研究对于其稳定可靠工 作非常重要。PARK ${ }^{\text {等 }}{ }^{[8]}$ 进行汽车双盘式磁流变液 制动器稳态温度场仿真。FALCÃO 等 ${ }^{[9]} \mathrm{c}^{2}$ 对一种以 水基型磁流变液为介质的车用磁流变液制动器, 开 展重复周期制动工况下温度场仿真。PATIL 等 ${ }^{[10]}$ 对 车用磁流变液制动器在城市交通工况下的温度特性 进行仿真分析。YU 等 ${ }^{[11]}$ 对车用磁流变液制动器进 行稳态温度仿真研究。郑军等 ${ }^{[12]}$ 开发了一种新型热 管式磁流变传动装置, 并测试了其散热性能。郑祥 盘等 ${ }^{[13]}$ 对曳引电梯用磁流变液制动装置不同工况 下的温度场进行有限元仿真。目前研究多未考虑汽 车实际驾驶过程中的不同制动工况需求, 并且主要 采用仿真方法, 缺乏系统的理论和试验支撑。

本文首先建立汽车制动过程中磁流变液制动器 的温度场数学模型, 其次针对汽车正常制动、紧急 制动和频繁间歇制动等工况, 进行瞬态温度分布及 其变化规律仿真分析; 最后搭建试验平台对其输出 制动力、制动性能和温度特性等进行试验研究, 以 期揭示其温度场分布及变化规律, 并为后续制动力 精确控制等研究提供仿真和试验依据。

\section{1 温度场数学模型}

在磁流变液制动器中任取一微元六面体, 由傅 里叶定律可知, 某一时刻 $t$ 流入该微元体左、右, 后、前和下、上侧表面的热流量分别为

$$
\left\{\begin{array}{l}
Q_{x}=-\lambda_{x} \frac{\partial t}{\partial x} \mathrm{~d} y \mathrm{~d} z, Q_{x+\mathrm{d} x}=Q_{x}+\frac{\partial}{\partial x}\left(-\lambda_{x} \frac{\partial t}{\partial x} \mathrm{~d} y \mathrm{~d} z\right) \mathrm{d} x \\
Q_{y}=-\lambda_{y} \frac{\partial t}{\partial y} \mathrm{~d} x \mathrm{~d} z, Q_{y+\mathrm{d} y}=Q_{y}+\frac{\partial}{\partial y}\left(-\lambda_{y} \frac{\partial t}{\partial y} \mathrm{~d} x \mathrm{~d} z\right) \mathrm{d} y(1) \\
Q_{z}=-\lambda_{z} \frac{\partial t}{\partial z} \mathrm{~d} x \mathrm{~d} y, Q_{z+\mathrm{d} z}=Q_{z}+\frac{\partial}{\partial z}\left(-\lambda_{z} \frac{\partial t}{\partial z} \mathrm{~d} x \mathrm{~d} y\right) \mathrm{d} z
\end{array}\right.
$$

式中, $\lambda_{x} 、 \lambda_{y} 、 \lambda_{z}$ 为微元体在 $x 、 y 、 z$ 方向上导 热系数, $\mathrm{d} x 、 \mathrm{~d} y 、 \mathrm{~d} z$ 为微元体的长、宽、高。

磁流变液制动器的瞬态温度场是关于坐标系和 时间的函数, 则在直角坐标系下微元体瞬态导热微 分方程为

$$
\begin{aligned}
\rho_{\mathrm{m}} c_{\mathrm{m}} \frac{\partial T_{\mathrm{m}}}{\partial t}= & \frac{\partial}{\partial x}\left(\lambda_{x} \frac{\partial T_{\mathrm{m}}}{\partial x}\right)+\frac{\partial}{\partial y}\left(\lambda_{y} \frac{\partial T_{\mathrm{m}}}{\partial y}\right)+ \\
& \frac{\partial}{\partial z}\left(\lambda_{z} \frac{\partial T_{\mathrm{m}}}{\partial z}\right)+\dot{\Phi}_{\mathrm{v}}
\end{aligned}
$$

式中, $\rho_{\mathrm{m}}$ 为微元体材料密度, $c_{\mathrm{m}}$ 为微元体比热容, $T_{\mathrm{m}}$ 为微元体温度, $\dot{\Phi}_{\mathrm{V}}$ 为微元体单位体积发热功率。

磁流变液制动器的热源主要包括磁流变液剪切 发热和线圈电功率发热。在汽车制动过程中, 磁流 变液剪切发热是制动器温升的主要原因, 它来自于 汽车行驶动能的损失量。在 $t$ 时刻、 $\Delta t$ 时间段内, 汽车行驶动能减少量 $\Delta E(t)$ 和动能损失功率 $P_{\mathrm{m}}(t)$ 的计算公式如下 ${ }^{[11]}$

$$
\left\{\begin{array}{l}
\Delta E(t)=\frac{1}{2} m\left\{\left(V_{0}-a t\right)^{2}-\left[V_{0}-a(t+\Delta t)\right]^{2}\right\} \\
P_{\mathrm{m}}(t)=\Delta E(t) / \Delta t=m a\left(V_{0}-a t\right)
\end{array}\right.
$$

式中, $V_{0}$ 为制动初速度, $a$ 为制动减速度, $m$ 为制 动器所处位置车辆质量, $m=\frac{G_{\mathrm{a}}\left(l-0.45 h_{\mathrm{g}}\right)}{2 L}$, 其中 $G_{\mathrm{a}}$ 是车辆满载总质量, $L$ 是车辆轴距, $h_{\mathrm{g}}$ 是车辆满 载时重心高度, $l$ 是重心至前轴距离。

线圈的电功率损失计算公式如下

$$
P_{\mathrm{c}}=I^{2} R_{\mathrm{c}}
$$

式中, $I$ 为线圈电流, $R_{\mathrm{c}}$ 为线圈电阻, $R_{\mathrm{c}}=\frac{N \pi \bar{d} \rho_{\mathrm{c}}}{S_{\mathrm{c}}}$, 其中 $N$ 为线圈匝数, $\bar{d} 、 \rho_{\mathrm{c}} 、 S_{\mathrm{c}}$ 分别为线圈平均 直径、导线电阻率和横截面积。

基于牛顿冷却定律, 制动过程中磁流变液制动 器与外界的换热功率可表示为

$$
P_{\mathrm{a}}=h_{\mathrm{a}} A_{\mathrm{s}}\left(T_{\mathrm{s}}-T_{\mathrm{e}}\right)
$$

式中, $h_{\mathrm{a}}$ 为对流换热系数, $A_{\mathrm{s}}$ 为换热面积, $T_{\mathrm{s}}$ 为壳 体外表面温度, $T_{\mathrm{e}}$ 为周围环境温度。

因此, 能量守恒方程的功率表示形式为

$$
P_{\mathrm{m}}+P_{\mathrm{c}}-P_{\mathrm{a}}=\sum c_{\mathrm{i}} \cdot m_{\mathrm{i}} \cdot \mathrm{d} T_{i} / \mathrm{d} t
$$

式中, $c_{\mathrm{i}} 、 m_{\mathrm{i}} 、 \mathrm{~d} T_{i} / \mathrm{d} t$ 分别为磁流变液制动器各部 分的比热容、质量和温度变化率。

\section{2 温度场仿真模型}

\section{1 研究对象}

考虑到汽车实际制动工况下的制动力矩需求, 所设计的磁流变液制动器采用多盘传动形式, 包括 4 对主动盘和制动盘, 相邻两盘间的工作间隙内充 满磁流变液, 其厚度为 $1.5 \mathrm{~mm}$ 。线圈未通电时, 磁 流变液表现为自由流动的牛顿流体状态; 当线圈通 
电后, 则在工作间隙内产生磁场, 磁流变液转变为 半固体状态, 从而产生制动力矩。本文在对其进行 温度场仿真时, 为提高计算速度, 忽略键槽、螺栓、 轴承、密封圈等影响, 图 1 所示为磁流变液制动器 结构简化模型剖视图。

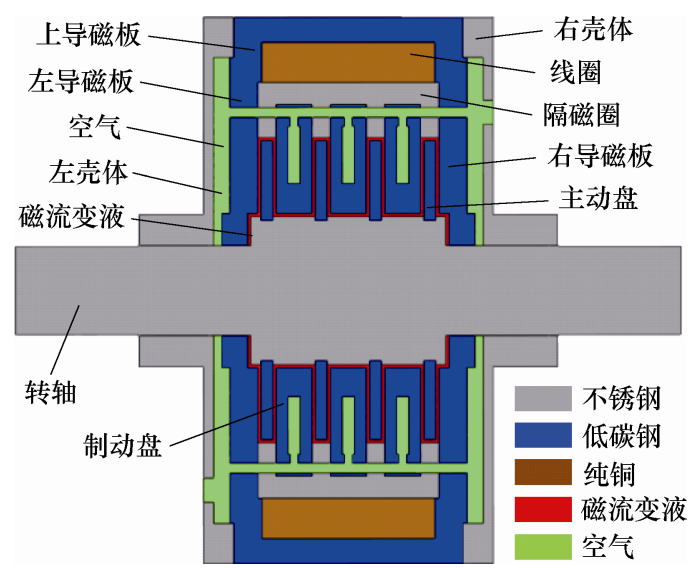

图 1 多盘式磁流变液制动器结构简化模型剖视图

\section{2 热物性参数}

所设计磁流变液制动器的材料热物性参数如表 1 所示。其中不锈钢 $0 \mathrm{Cr} 18 \mathrm{Ni}$ 、20 钢、纯铜 $\mathrm{Cu}$ 的 热物性参数由热工手册中查询得到。选用的磁流变 液 MRF-J01 购自宁波杉工智能安全科技股份有限 公司，其热物性参数由厂商提供。

\section{表 1 制动器各部分材料的热物性参数表}

\begin{tabular}{lccc}
\hline \multicolumn{1}{c}{ 材料 } & $\begin{array}{c}\text { 热导率 } \\
\lambda /\left(\mathrm{W} \cdot \mathrm{m}^{-1} \cdot \mathrm{K}^{-1}\right)\end{array}$ & $\begin{array}{c}\text { 密度 } \\
\rho /\left(\mathrm{kg} \cdot \mathrm{m}^{-3}\right)\end{array}$ & $\begin{array}{c}\text { 比热容 } \\
c /\left(\mathrm{J} \cdot \mathrm{kg}^{-1} \cdot \mathrm{K}^{-1}\right)\end{array}$ \\
\hline 不锈钢 $0 \mathrm{Cr} 18 \mathrm{Ni9}$ & 14 & 7900 & 510 \\
20 钢 & 48 & 7850 & 480 \\
纯铜 $\mathrm{Cu}$ & 393 & 8900 & 390 \\
磁流变液 MRF-J01 & 1 & 3090 & 1000 \\
\hline
\end{tabular}

\section{3 有限元网格划分}

基于磁流变液制动器结构和热物性参数, 对其 进行网格划分。选用四面体网格单元, 划分方式为 自由网格划分。此外, 对磁流变液处的网格进行适 当加密以提高计算精度。磁流变液制动器有限元网 格划分结果如图 2 所示。
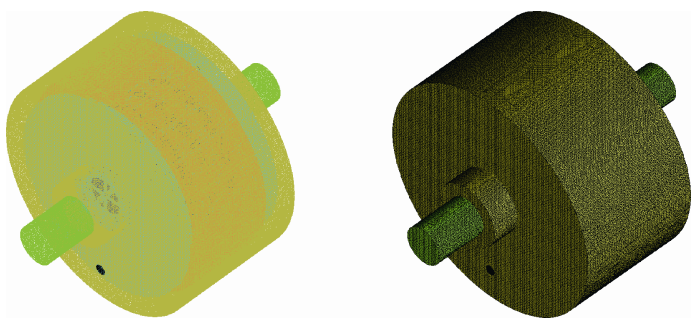

图 2 磁流变液制动器有限元模型

\section{4 热载荷及边界条件设置}

将制动器初始温度和环境温度均设定为 $25{ }^{\circ} \mathrm{C}$,
施加的热载荷包括：线圈区域和各个工作间隙区域 的生热率、制动器与外界环境间的对流换热和热辐 射。热载荷及边界条件可以表示为常量或函数表达 式, 其中线圈区域的生热率采用常量输入、各个工 作间隙区域的生热率采用函数表达式输入。

假定汽车制动时的动能损失全部转换为工作 间隙热量，则各个工作间隙区域的生热率 $\phi_{\mathrm{m}}$ 可表 示为

$$
\phi_{\mathrm{m}}=P_{\mathrm{m}} / V_{\mathrm{m}}
$$

式中， $V_{\mathrm{m}}$ 为工作间隙中磁流变液体积。

假定制动器工作时线圈的电功率损失全部用于 线圈区域的温升, 则线圈区域的生热率 $\phi_{\mathrm{c}}$ 为

$$
\phi_{\mathrm{c}}=P_{\mathrm{c}} / V_{\mathrm{c}}
$$

式中, $V_{\mathrm{c}}$ 为线圈体积, $V_{\mathrm{c}}=\pi b_{\mathrm{c}}\left(r_{\mathrm{c}_{2}}^{2}-r_{\mathrm{c}_{1}}^{2}\right)$, 其中 $r_{\mathrm{c}_{1}}$ 、 $r_{\mathrm{c}_{2}}$ 分别为线圈内、外半径, $b_{\mathrm{c}}$ 为线圈高度。

静止壳体外表面与周围空气之间同时存在自然 对流换热和辐射换热，其复合换热系数可表示为

$$
\partial_{\mathrm{s}}=\partial_{\mathrm{c}}+\partial_{\mathrm{r}}
$$

式中, $\partial_{\mathrm{c}}$ 为自然对流换热系数, $\partial_{\mathrm{r}}$ 为辐射换热系数。 根据文献 $[14], \quad \partial_{\mathrm{s}}=9.7 \mathrm{~W} \cdot \mathrm{m}^{-2} \cdot{ }^{\circ} \mathrm{C}^{-1}$ 。

转轴外表面和端面与周围空气同时存在强制对 流换热和辐射换热，其换热系数可表示为

$$
\partial_{\mathrm{k}}=28\left(1+\sqrt{\frac{0.45 \pi \bar{n}_{\mathrm{s}} d_{\mathrm{s}}}{60}}\right)
$$

式中, $d_{\mathrm{s}}$ 为旋转面平均直径, $\bar{n}_{\mathrm{s}}$ 为转轴平均转速。

根据汽车制动器实际应用中对制动时间和制动 减速度的要求, 选取图 3 中的正常制动、紧急制动、 频繁间歇制动等三种不同制动工况进行研究。其中, 正常制动(工况一)的制动减速度为 $3 \mathrm{~m} / \mathrm{s}^{2}$, 紧急制动 (工况二)的制动减速度为 $7.5 \mathrm{~m} / \mathrm{s}^{2}$, 频繁间歇制动(工 况三)的制动减速度为: $1 \sim 3 \mathrm{~s}$ 为 $3.5 \mathrm{~m} / \mathrm{s}^{2} 、 5 \sim 8 \mathrm{~s}$ 为 $2 \mathrm{~m} / \mathrm{s}^{2} 、 10 \sim 13 \mathrm{~s}$ 为 $2 \mathrm{~m} / \mathrm{s}^{2} 、 15.0 \sim 16.6 \mathrm{~s}$ 为 $2 \mathrm{~m} / \mathrm{s}^{2}$ 。

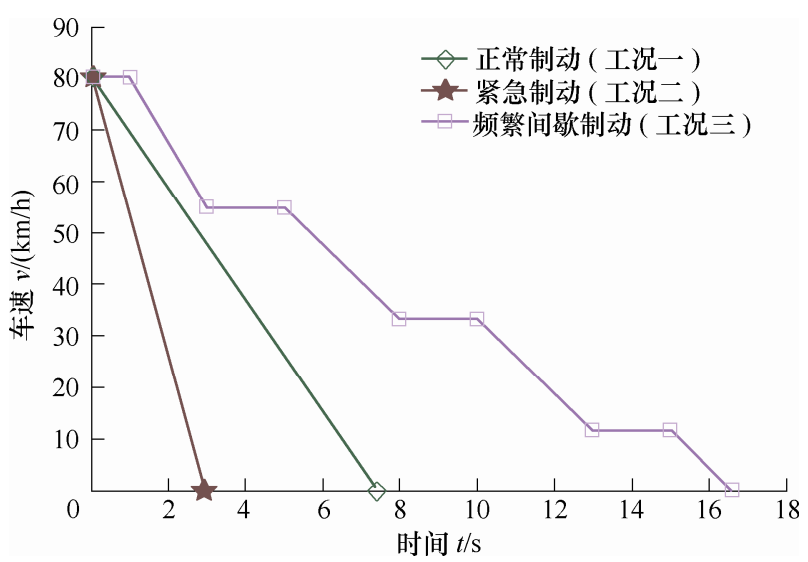

图 3 不同制动工况下车速随时间的变化关系 
图 4 表示各制动工况下工作间隙生热率随时间的变 化关系。仿真时, 制动器热载荷和边界条件的具体 数值如表 2 所示。

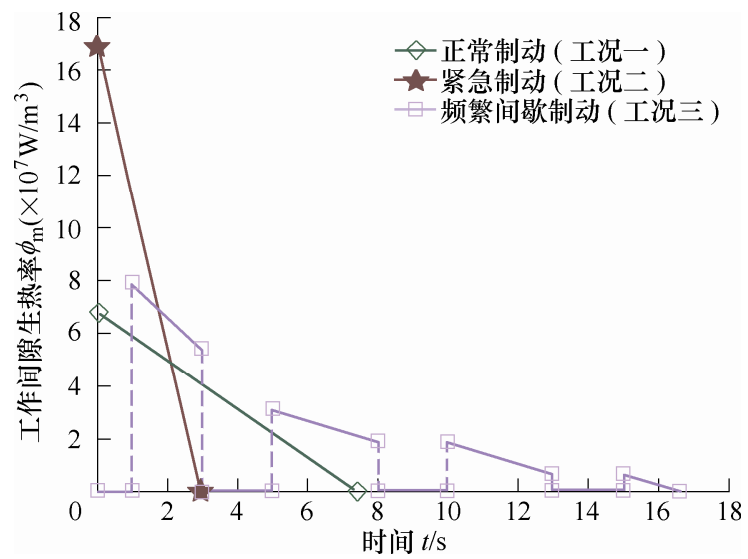

图 4 不同制动工况下工作间隙生热率随时间的变化关系

表 2 热载荷和边界条件设置参数表

\begin{tabular}{|c|c|c|c|c|}
\hline \multirow{2}{*}{ 区域 } & \multirow{2}{*}{ 热载荷和边界条件参数 } & \multicolumn{3}{|c|}{ 参数值 } \\
\hline & & 工况一 & 工况二 & 工况三 \\
\hline \multirow{2}{*}{ 线圈 } & 发热功率 $P_{\mathrm{c}} / \mathrm{kW}$ & 0.032 & 0.152 & 0.032 \\
\hline & 生热率 $\varphi_{\mathrm{c}} /\left(\mathrm{W} \cdot \mathrm{m}^{-3}\right)$ & $2.59 \times 10^{4}$ & $1.23 \times 10^{5}$ & $2.59 \times 10^{4}$ \\
\hline \multirow{2}{*}{ 工作间隙 } & 动能损失功率 $P_{\mathrm{m}} / \mathrm{kW}$ & \multicolumn{3}{|c|}{$P_{\mathrm{m}}=m a\left(V_{0}-a t\right)$} \\
\hline & 生热率 $\varphi_{\mathrm{m}} /\left(\mathrm{W} \cdot \mathrm{m}^{-3}\right)$ & \multicolumn{3}{|c|}{$\varphi_{\mathrm{m}}=P_{\mathrm{m}} / V_{\mathrm{m}}$} \\
\hline \multirow{2}{*}{$\begin{array}{c}\text { 静止壳体 } \\
\text { 表面 }\end{array}$} & 环境温度 $T_{\mathrm{e}}{ }^{\circ} \mathrm{C}$ & \multicolumn{3}{|c|}{25} \\
\hline & 换热系数 $\partial_{\mathrm{s}} /\left(\mathrm{W} \cdot \mathrm{m}^{-2} \cdot{ }^{\circ} \mathrm{C}^{-1}\right)$ & \multicolumn{3}{|c|}{9.7} \\
\hline \multirow{2}{*}{$\begin{array}{l}\text { 转轴外表 } \\
\text { 面和端面 }\end{array}$} & 平均转速 $\bar{n}_{\mathrm{S}} /\left(\mathrm{r} \cdot \mathrm{min}^{-1}\right)$ & \multicolumn{3}{|c|}{442} \\
\hline & 换热系数 $\partial_{\mathrm{K}} /\left(\mathrm{W} \cdot \mathrm{m}^{-2} \cdot{ }^{\circ} \mathrm{C}^{-1}\right)$ & \multicolumn{3}{|c|}{45.61} \\
\hline
\end{tabular}

\section{3 仿真结果与分析}

基于上述温度场仿真模型, 以某款 $\mathrm{A} 0$ 级汽车 为研究对象, 其主要参数如表 3 所示。经过迭代计 算, 分别模拟出汽车处于三种不同制动工况下磁流 变液制动器的瞬态温度场并进行对比分析。

\section{表 3 某款 $\mathrm{A0}$ 级汽车主要参数表}

\begin{tabular}{cccccc}
\hline 参数 & $\begin{array}{c}\text { 满载总重量 } \\
G_{\mathrm{a}} / \mathrm{kg}\end{array}$ & $\begin{array}{c}\text { 轴距 } \\
L / \mathrm{m}\end{array}$ & $\begin{array}{c}\text { 满载时重心 } \\
\text { 高度 } h_{\mathrm{g}} / \mathrm{m}\end{array}$ & $\begin{array}{c}\text { 重心至前轴 } \\
\text { 距离 } l / \mathrm{m}\end{array}$ & $\begin{array}{c}\text { 车轮滚动 } \\
\text { 半径 } r_{\mathrm{f}} / \mathrm{m}\end{array}$ \\
\hline 数值 & 1150 & 2.34 & 0.55 & 1.14 & 0.24 \\
\hline
\end{tabular}

\section{1 正常制动工况下制动器瞬态温度场}

正常制动工况下, 经过一个制动周期得到制动 器温度分布云图如图 5 所示。可以看出, 制动器温 度最高的区域在内部主动盘处; 温度次高的区域位 于内部磁流变液和制动盘处。这主要是因为这些位 置处于制动器最内部, 其热阻较大导致散热性能较 其他区域稍差。

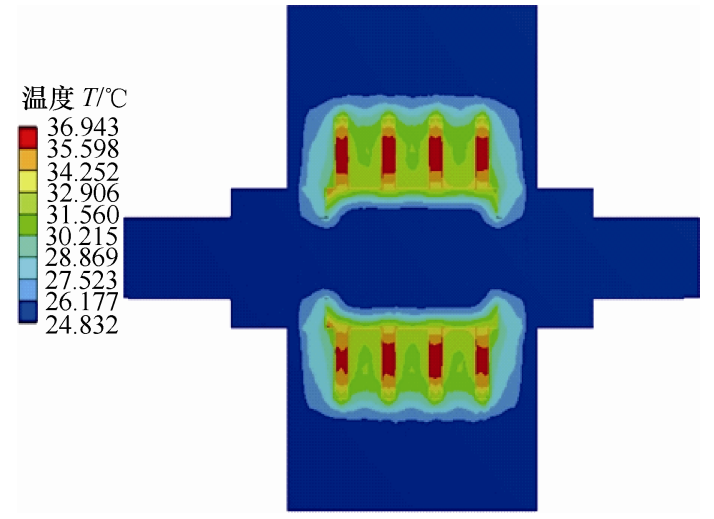

图 5 正常制动结束时磁流变液制动器温度分布云图

图 6 为一个制动周期内制动器最高温度随时间 变化曲线。从图中可以看出, 制动初期由于汽车动 能损失功率大于外界散热功率, 制动器最高温度随 时间呈现迅速上升趋势, 在 $3.11 \mathrm{~s}$ 时达到最高值 $45.4{ }^{\circ} \mathrm{C}$; 随后, 车速降低, 动能损失功率小于外界 散热功率, 最高温度逐渐下降并在一个制动周期完 成后达到 $36.9{ }^{\circ} \mathrm{C}$ 。

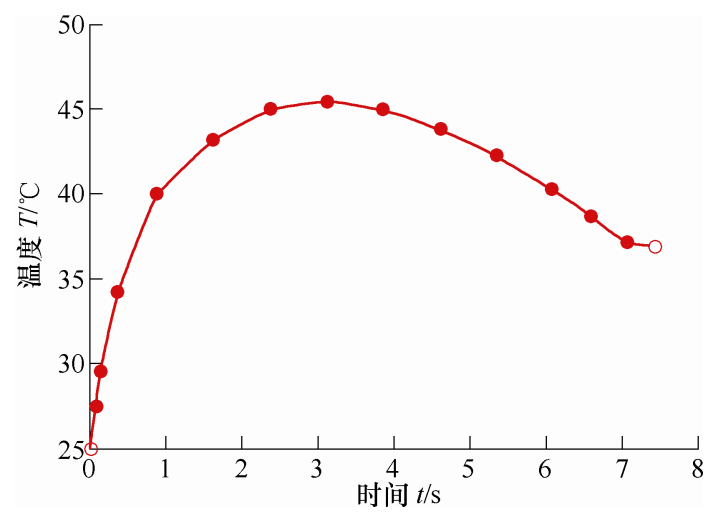

图 6 正常制动工况下最高温度随时间变化曲线

\section{2 紧急制动工况下制动器瞬态温度场}

紧急制动工况下，经过一个制动周期得到制动 器温度分布云图如图 7 所示。可以看出, 制动器最 高温度位于磁流变液处; 温度次高的区域位于制动 盘处。相比于正常制动工况, 紧急制动工况的温度

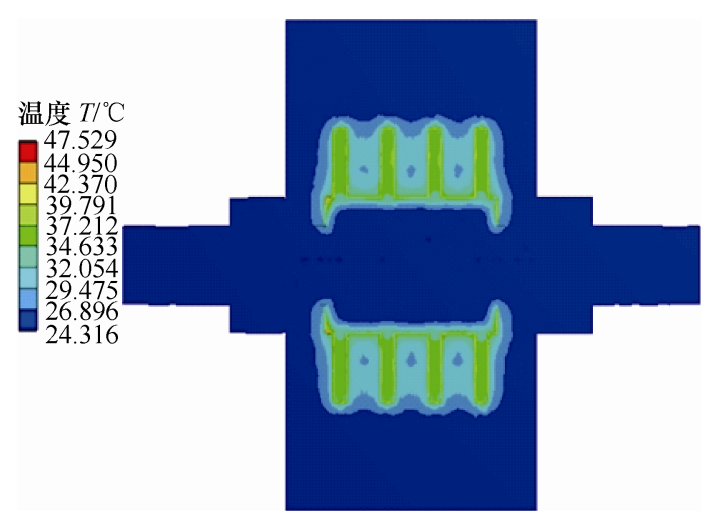

图 7 紧急制动结束时磁流变液制动器温度分布云图 
分布稍有不同。这是由于紧急制动周期短, 工作间 隙处产生的热量来不及向周围扩散, 导致磁流变液 处温度比正常制动工况要高。

图 8 为一个制动周期内制动器最高温度随时间 变化曲线。由图可见, 制动器内部最高温度同样随 时间呈现先迅速上升后缓慢下降的变化趋势, 但相 比于正常制动工况, 制动初期温度上升速度明显较 快。在 $1.30 \mathrm{~s}$ 时达到最高温度 $60.2{ }^{\circ} \mathrm{C}$, 并在一个制 动周期完成后下降至 $47.5{ }^{\circ} \mathrm{C}$ 。

\section{3 频繁间歇制动工况下制动器瞬态温度场}

频繁间歇制动工况下，经过一个制动周期得到 不同时刻制动器温度分布云图如图 9a 9f 所示。可 以看出, 在整个制动周期内制动器温度较高区域仍 然位于磁流变液和制动盘处。工作间隙处产生的热

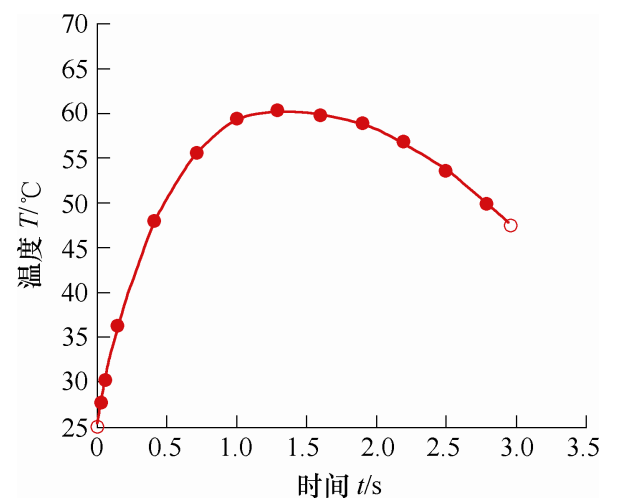

图 8 紧急制动工况下最高温度随时间变化曲线

量随时间不断向周围扩散，由于制动盘位于最内部 且被磁流变液包覆, 其热阻较大, 导致制动盘区域 温度高于其他部位。

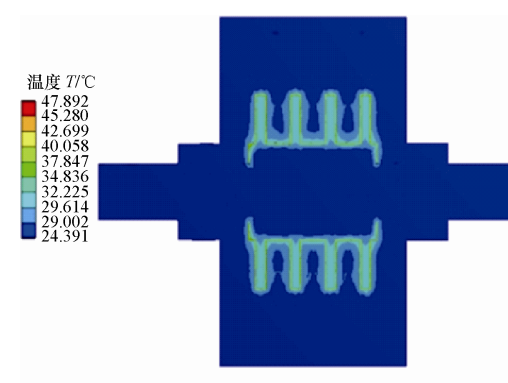

(a) $t=3 \mathrm{~s}$

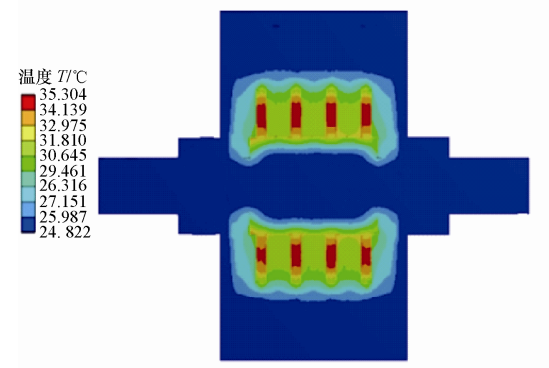

(d) $t=10 \mathrm{~s}$

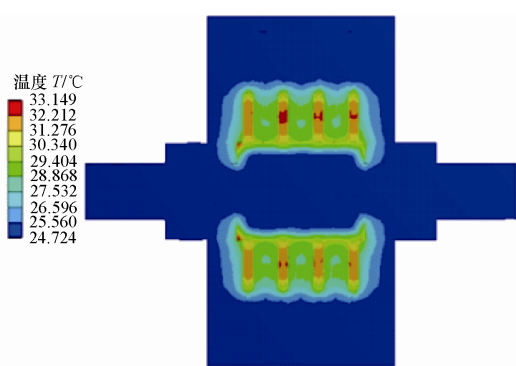

(b) $t=5 \mathrm{~s}$

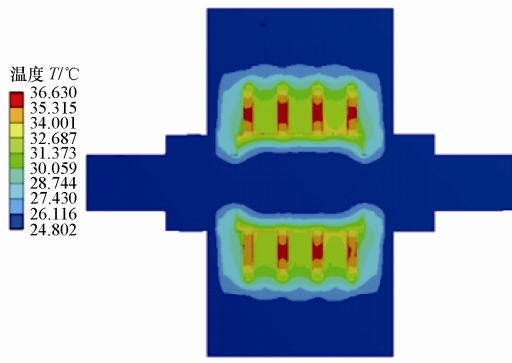

(e) $t=13 \mathrm{~s}$

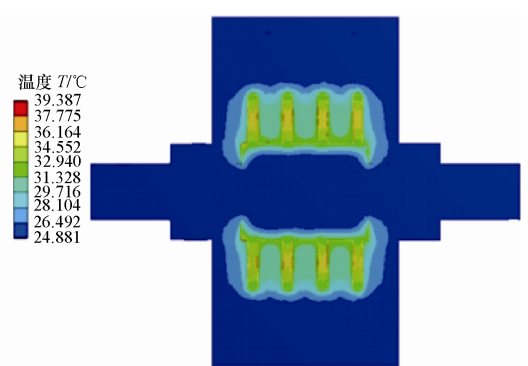

(c) $t=8 \mathrm{~s}$

图 9 频繁间歇制动工况下不同时刻磁流变液制动器温度分布云图

图 10 为一个制动周期内制动器最高温度随时 间变化曲线。当制动器介入制动时, 制动器内部工 作间隙处的温度逐渐上升; 当制动器停止工作时,

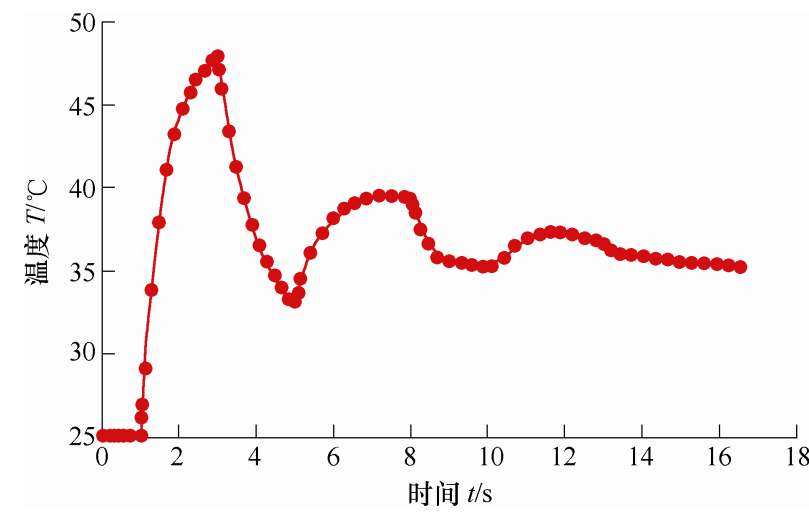

图 10 频繁间歇制动工况下最高温度随时间变化曲线
内部产生的热量向外扩散导致温度降低。具体而言, 在制动器介入制动结束的三个时刻 $3 \mathrm{~s} 、 8 \mathrm{~s}$ 和 $13 \mathrm{~s}$, 最高温度值分别为 $47.9{ }^{\circ} \mathrm{C} 、 39.4{ }^{\circ} \mathrm{C}$ 和 $36.6{ }^{\circ} \mathrm{C}$; 当 制动器停止工作一段时间后的三个时刻 $5 \mathrm{~s} 、 10 \mathrm{~s}$ 和 $16.6 \mathrm{~s}$ 时, 最高温度值则分别为降至 $33.1{ }^{\circ} \mathrm{C}$ 、 $35.3{ }^{\circ} \mathrm{C}$ 和 $35.2{ }^{\circ} \mathrm{C}$ 。

\section{4 试验测试}

试验平台模拟对象为 $\mathrm{A} 0$ 级汽车，采用单端惯 性飞轮模拟方式。图 11 所示为试验平台实物图, 主 要由驱动电动机、惯性飞轮组、转速转矩传感器、 多盘式磁流变液制动器、变频器、程控电源、稳压 电源 $(24 \mathrm{~V})$ 、上位机和数据采集组件等组成。根据 
乘用车制动器性能要求及台架试验方法 ${ }^{[15]}$, 惯性飞 轮组转动惯量计算公式如下

$$
I_{\mathrm{f}}=m r_{\mathrm{f}}^{2}
$$

式中, $r_{\mathrm{f}}$ 为车轮滚动半径。

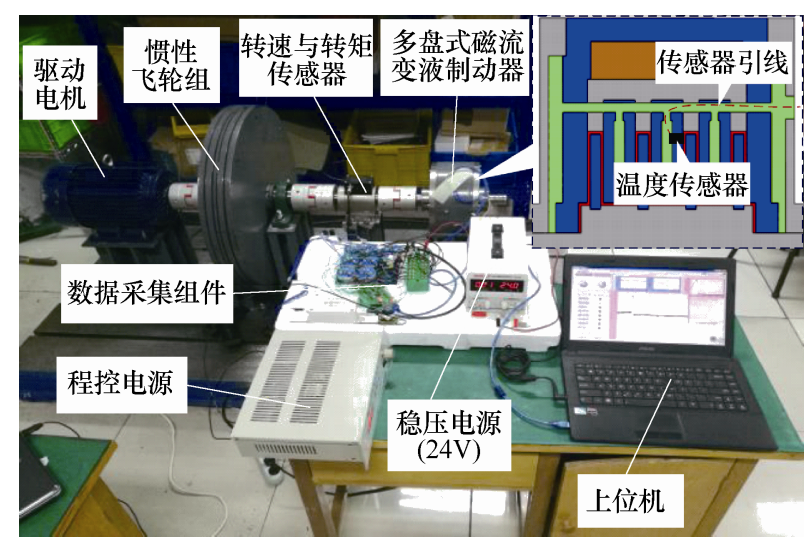

图 11 单端惯性飞轮式汽车磁流变液制动器试验平台

试验步骤如下: (1) 根据工况要求, 调节程控电 源输出电流值后将其关闭, 通过变频器调定制动初 速度; (2) 启动驱动电动机带动惯性飞轮组转动, 利 用飞轮高速旋转的能量模拟汽车行驶过程中所具有 的动能; (3) 当飞轮转速到达等效车速(对应车辆制 动初速度)时, 关闭电动机同时开启程控电源, 使磁 流变液制动器介入制动直至惯性飞轮组完全停止, 此为一个制动周期。

\section{1 磁流变液制动器输出制动力测试}

输出制动力测试即为恒定线圈电流条件下的制 动试验, 首先使飞轮转速达到等效车速, 再接通电 流进行制动直至飞轮停止, 即为一个制动周期。试 验中设定制动初速度为 $40 \mathrm{~km} / \mathrm{h}$, 线圈电流分别为

\section{$1.1 \mathrm{~A}$ 和 $2.4 \mathrm{~A}$ 。}

图 12 为磁流变液制动器输出制动力随时间变 化曲线。由图可见, 刚开始时输出制动力随线圈电 流的接通而阶跃上升, 随着制动周期逐渐临近结束, 输出制动力略有降低, 这是由于制动器转速降低导

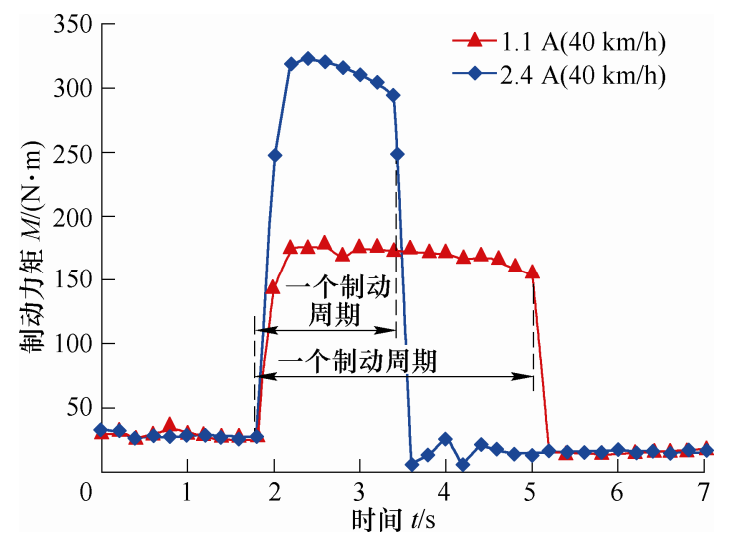

图 12 磁流变液制动器输出制动力随时间变化曲线
致磁流变液黏性阻力减小所致。当电流为 $1.1 \mathrm{~A}$ 时, 制动器最大输出制动力为 $176.9 \mathrm{~N} \cdot \mathrm{m}$; 当电流增至 $2.4 \mathrm{~A}$, 最大输出制动力达到 $321.9 \mathrm{~N} \cdot \mathrm{m}$ 。

\section{2 磁流变液制动器制动性能测试}

制动性能测试即为不同制动初速度和线圈电流 条件下的制动试验。试验中选定制动初速度分别为 $40 \mathrm{~km} / \mathrm{h} 、 60 \mathrm{~km} / \mathrm{h}$ 和 $80 \mathrm{~km} / \mathrm{h}$, 线圈电流分别为 $1.1 \mathrm{~A}$ 和 $2.4 \mathrm{~A}$ 。

图 13 为线圈电流为 $1.1 \mathrm{~A}$ 和 $2.4 \mathrm{~A}$ 时, 不同制 动初速度条件下一个制动周期内车速试验值与计算 值对比情况。可以看出，在相同电流下，一个制动 周期内制动减速度基本保持不变，这表明所设计的 汽车磁流变液制动器具有良好的恒减速度制动特 性。但在制动周期临近结束时, 由于制动器输出制 动力略为下降, 制动减速度稍有减小。具体而言, 当电流分别为 $1.1 \mathrm{~A}$ 和 $2.4 \mathrm{~A}$ 时, 一个制动周期内的 制动减速度平均值分别为 $2.96 \mathrm{~m} / \mathrm{s}^{2}$ 和 $7.39 \mathrm{~m} / \mathrm{s}^{2}$, 满 足汽车实际制动工况需求。

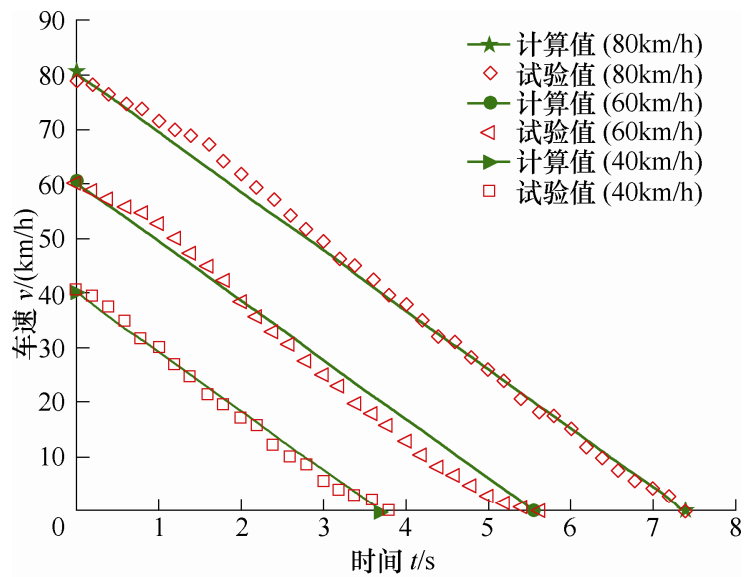

(a) 线圈电流 $1.1 \mathrm{~A}$

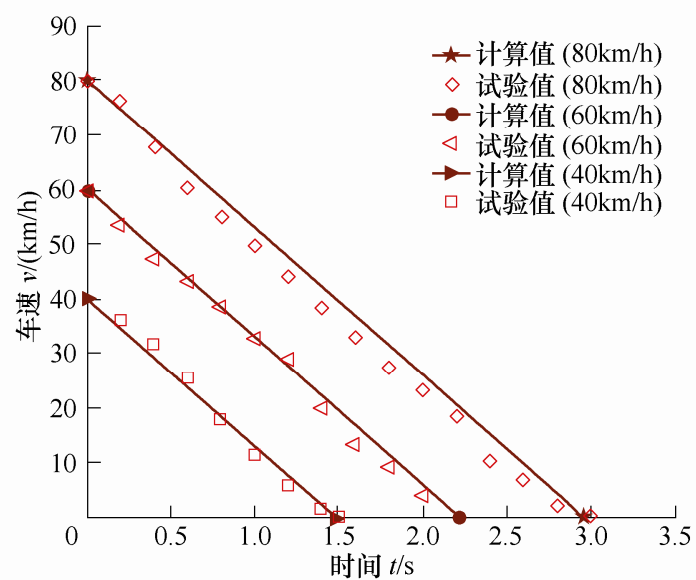

(b) 线圈电流 $2.4 \mathrm{~A}$

图 13 不同制动初速度条件下一个制动周期内车速 试验值与计算值对比情况

\section{3 磁流变液制动器温度特性测试}

温度特性测试即通过采集不同制动初速度和不 
同线圈电流条件下温度传感器信号, 以获得一个制 动周期内制动器内部温度变化规律。试验中选定制 动初速度分别为 $40 \mathrm{~km} / \mathrm{h} 、 60 \mathrm{~km} / \mathrm{h}$ 和 $80 \mathrm{~km} / \mathrm{h}$, 线 圈电流分别为 $1.1 \mathrm{~A}$ 和 $2.4 \mathrm{~A}$ 。

图 14 为线圈电流为 $1.1 \mathrm{~A}$ 和 $2.4 \mathrm{~A}$ 时, 不同制 动初速度条件下一个制动周期内测点处温度随时间 变化规律。由图 14 可见, 测点处温升随着制动初速 度和线圈电流的增加而显著增大; 当电流为 $2.4 \mathrm{~A}$ 、 制动初速度分别为 $40 \mathrm{~km} / \mathrm{h} 、 60 \mathrm{~km} / \mathrm{h}$ 和 $80 \mathrm{~km} / \mathrm{h}$ 时, 一个制动周期内测点处最高温升分别为 $16.3{ }^{\circ} \mathrm{C}$ 、 $19.5{ }^{\circ} \mathrm{C}$ 和 $30.3{ }^{\circ} \mathrm{C}$, 由于试验中选用的 MRF-J01 型 磁流变液许用工作温度范围为 $-40 \sim 130{ }^{\circ} \mathrm{C}$, 表明其 符合磁流变液工作温度要求。此外, 通过对比工况 一和工况二测点处温度的仿真值和试验值可知, 两 者在数值和趋势上较为吻合, 最大差值分别为 $4.7{ }^{\circ} \mathrm{C}$ (工况一)和 $6.5{ }^{\circ} \mathrm{C}$ (工况二), 考虑到仿真时的 模型简化、周围环境中换热和对流的差异以及传感 器测试精度等一系列因素影响, 表明本文中所建立 的汽车磁流变液制动器温度场仿真模型能够较好地 反映其实际制动工作过程的温度特性。

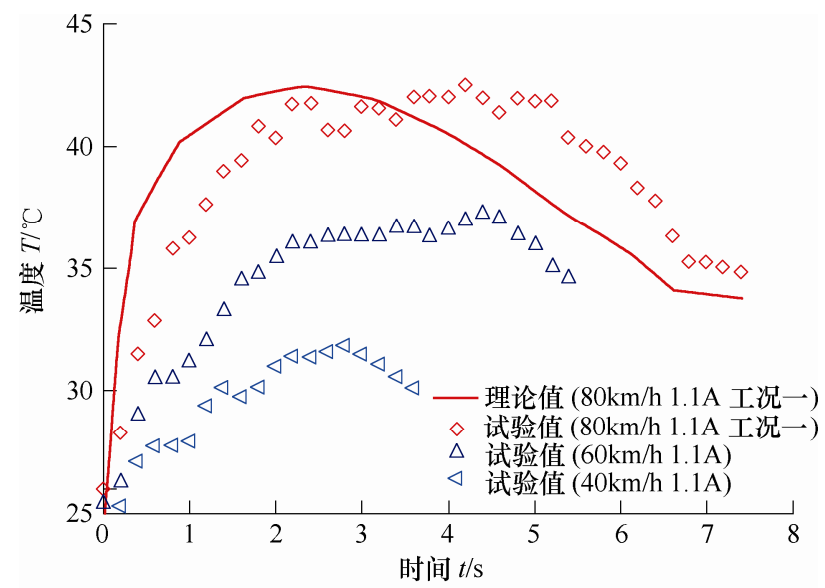

(a) 线圈电流 $1.1 \mathrm{~A}$

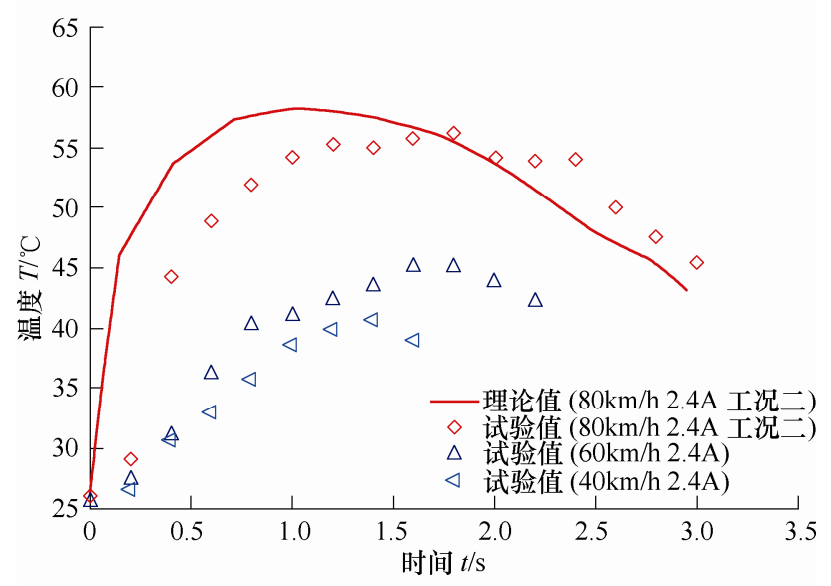

(b) 线圈电流 $2.4 \mathrm{~A}$

图 14 不同制动初速度条件下一个制动周期内测点处 温度随时间变化曲线

\section{5 结论}

（1）针对磁流变液制动器的制动性能试验表 明，其产生的制动减速度的实测值与计算值基本一 致; 在相同电流下，一个制动周期内制动减速度基 本维持恒定, 呈现出良好的恒减速度制动特性; 当 电流为 $1.1 \mathrm{~A}$ 和 $2.4 \mathrm{~A}$ 时, 实测制动减速度分别约为 $2.96 \mathrm{~m} / \mathrm{s}^{2}$ 和 $7.29 \mathrm{~m} / \mathrm{s}^{2}$, 满足汽车实际制动工况需求。

(2) 当汽车处于正常制动、紧急制动和频繁间 隙制动等三种工况下, 分别对磁流变液制动器的瞬 态温度特性进行仿真分析, 并通过试验进行了验证。 结果表明，工作间隙内温度呈现先迅速增大后逐渐 降低的变化过程, 并且制动初速度和线圈电流越大, 温升幅度和速率均会增大; 在制动初速度 $80 \mathrm{~km} / \mathrm{h}$ 、 制动减速度 $7.39 \mathrm{~m} / \mathrm{s}^{2}$ 的紧急制动工况下，一个制动 周期内实测最高温升为 $30.3{ }^{\circ} \mathrm{C}$, 符合所选磁流变液 工作温度要求。

(3) 通过对比整个制动周期内制动器测点处温 度的试验值与仿真值, 发现两者在数值和趋势上较 为吻合，最大差值为 $6.5{ }^{\circ} \mathrm{C}$, 考虑到仿真时的模型 简化、周围环境中换热和对流的差异以及传感器测 试精度等一系列因素影响, 表明所建立的温度场仿 真模型能够较好地反映磁流变液制动器实际制动过 程的温度特性。

\section{参 考 文 献}

[1] WAGNER A , SPELSBERG-KORSPETER G , HAGEDORN P. Structural optimization of an asymmetric automotive brake disc with cooling channels to avoid squeal[J]. Journal of Sound and Vibration, 2014, 333(7): 1888-1898.

[2] WANG N, WANG S, PENG Z, et al. Braking control performances of a disk-type magneto-rheological brake via hardware-in-the-loop simulation[J]. Journal of Intelligent Material Systems and Structures, 2018, 29(20): 3937-3948.

[3] 孔祥东, 李斌, 权凌霄, 等. 磁流变液阻尼器 Bingham多项式力学模型研究 [J]. 机械工程学报, 2017, 53(14): 179-186.

KONG Xiangdong, LI Bin, QUAN Lingxiao, et al. Study on dynamic bingham-polynomial model of a MRF damper[J]. Journal of Mechanical Engineering, 2017, 53(14): 179-186.

[4] KEREM K , EDWARD J P , AFZAL S. Design considerations for an automotive magnetorheological brake[J]. Mechatronics, 2008, 18(8): 434-447. 
[5] ASSADSANGABI B, DANESHMAND F, VAHDATI N, et al. Optimization and design of disk-type MR brakes[J]. International Journal of Automotive Technology, 2011, 12(6): 921-932.

[6] WANG Daoming, HOU Youfu, TIAN Zuzhi. A novel high-torque magnetorheological brake with a water cooling method for heat dissipation[J]. Smart Materials and Structures, 2013, 22(2): 025019.

[7] WANG Daoming, ZI Bin, ZENG Yishan, et al. An investigation of thermal characteristics of a liquid-cooled magnetorheological fluid-based clutch[J]. Smart Materials and Structures, 2015，24(5): 055020.

[8] PARK E J, STOIKOV D, FALCÃO da Luz L, et al. A performance evaluation of an automotive magnetorheological brake design with a sliding mode controller[J]. Mechatronics, 2006, 16(7): 405-416.

[9] FALCÃO da Luz L. Design of a magnetorheological brake system[D]. Canada: University of Victoria, 2004.

[10] PATIL S R, POWAR K P, SAWANT S M. Thermal analysis of magnetorheological brake for automotive application[J]. Applied Thermal Engineering, 2016, 98: 238-245.

[11] YU Liangyao, MA Liangxu, SONG Jian, et al. Magnetorheological and wedge mechanism-based brake-by-wire system with self-energizing and self-powered capability by brake energy harvesting[J]. IEEE/ASME Transactions on Mechatronics, 2016, 21(5): 2568-2580.
[12] 郑军, 张光辉, 曹兴进. 热管式磁流变传动装置的设计 与试验[J]. 机械工程学报, 2009, 45(7): 305-311.

ZHENG Jun, ZHANG Guanghui, CAO Xingjin. Design and experiment for magnetorheological transmission device with heat pipes[J]. Journal of Mechanical Engineering, 2009, 45(7): 305-311.

[13] 郑祥盘, 陈凯峰, 陈淑梅. 曳引电梯磁流变制动装置的 温度特性研究 $[\mathrm{J}]$. 中国机械工程, 2016, 27(16)： 2141-2147.

ZHENG Xiangpan, CHEN Kaifeng, CHEN Shumei. Investigation on temperature properties of elevator magnetorheological brake[J]. China Mechanical Engineering, 2016, 27(16): 2141-2147.

[14] 丁舜年. 大型电机的发热与冷却 $[\mathrm{M}]$. 北京: 科学出版 社, 1992.

DING Shunnian. Heating and cooling of large motor[M]. Beijing: Science Press, 1992.

[15] 全国汽车标准化技术委员会. QC/T564-2008 乘用车制 动器性能要求及台架试验方法[S]. 北京: 中国计划出版 社, 2008.

National Technical Committee of Auto Standardization. QC/T564-2008 Performance requirements and bench test methods for passenger car brake[S]. Beijing: China Planning Press, 2008.

作者简介: 王道明(通信作者), 男, 1987 年出生, 博士, 副教授。主要 研究方向为智能结构与系统、磁流变技术及应用和车辆先进制动技术。 E-mail: denniswang@hfut.edu.cn 